\title{
The effect of virtual reality on temporal bone anatomy evaluation and performance
}

\author{
Tomi Timonen ${ }^{1,5} \cdot$ Aarno Dietz $^{1,5}$ (1) $\cdot$ Pia Linder $^{1}$ (1) $\cdot$ Antti Lehtimäki ${ }^{4} \cdot$ Heikki Löppönen ${ }^{1,5}$. \\ Antti-Pekka Elomaa ${ }^{2,3}$ (1) $\cdot$ Matti Iso-Mustajärvi ${ }^{1,2}$ (1)
}

Received: 10 August 2021 / Accepted: 15 November 2021 / Published online: 27 November 2021

(c) The Author(s) 2021

\begin{abstract}
Purpose There is only limited data on the application of virtual reality (VR) for the evaluation of temporal bone anatomy. The aim of the present study was to compare the VR environment to traditional cross-sectional viewing of computed tomography images in a simulated preoperative planning setting in novice and expert surgeons.

Methods A novice $(n=5)$ and an expert group $(n=5)$, based on their otosurgery experience, were created. The participants were asked to identify 24 anatomical landmarks, perform 11 distance measurements between surgically relevant anatomical structures and 10 fiducial markers on five cadaver temporal bones in both VR environment and cross-sectional viewings in PACS interface. The data on performance time and user-experience (i.e., subjective validation) were collected.

Results The novice group made significantly more errors $(p<0.001)$ and with significantly longer performance time $(p=0.001)$ in cross-sectional viewing than the expert group. In the VR environment, there was no significant differences (errors and time) between the groups. The performance of novices improved faster in the VR. The novices showed significantly faster task performance $(p=0.003)$ and a trend towards fewer errors $(p=0.054)$ in VR compared to cross-sectional viewing. No such difference between the methods were observed in the expert group. The mean overall scores of userexperience were significantly higher for VR than cross-sectional viewing in both groups $(p<0.001)$.

Conclusion In the VR environment, novices performed the anatomical evaluation of temporal bone faster and with fewer errors than in the traditional cross-sectional viewing, which supports its efficiency for the evaluation of complex anatomy.
\end{abstract}

Keywords Virtual reality $\cdot$ Surgery training $\cdot$ Surgical planning $\cdot$ Temporal bone $\cdot$ Anatomy training

\section{Introduction}

Historically, learning of anatomy has relied on traditional methods, including two-dimensional (2D) image representations (e.g., anatomy textbooks, pictures, and radiological

Tomi Timonen

tomi.timonen@kuh.fi

1 Department of Otorhinolaryngology, Kuopio University Hospital, Puijonlaaksontie 2, 70210 Kuopio, PL 100, 70029 Kuopio, Finland

2 Microsurgery Centre of Eastern Finland, Kuopio, Finland

3 Department of Neurosurgery, Kuopio University Hospital, Kuopio, Finland

4 Department of Radiology, Kuopio University Hospital, Kuopio, Finland

5 School of Medicine, Institute of Clinical Medicine, University of Eastern Finland, Kuopio, Finland images), cadaver dissections and assisting or observing during live operations. Medical students, residents and young surgeons often experience difficulties in obtaining a wellgrounded grasp of three-dimensional (3D) anatomy from 2D images and illustrations [1], and the inadequacy of resources for cadaver dissections and live operations may become a hindrance for adequate 3D understanding $[2,3]$.

Virtual reality (VR) technology offers promising stereoscopic applications for the teaching of anatomy, surgical training and preoperative planning [4, 5]. Previous studies have supported the benefits of anatomical VR visualization methods over traditional 2D methods for adding to students' knowledge of factual anatomy [6, 7]. However, there is still a lack of clinical evidence of the impact of VR environments on learning anatomical features and achieving a contextual understanding of dimensional relationships [8-10].

Even though the new medical imaging techniques provide high resolution in each dimension, image data are still 
often examined as a series of 2D cross-sectional images [11]. The reduced costs, advances in computational performance and availability of consumer-grade VR technology have increased interest in medical applications of VR. It has been proposed that VR applications designed for anatomy education and surgical planning may prove themselves beneficial through the improved 3D perception of complex structures $[12,13]$. In principle, VR technology can provide a 3D computer-generated environment, which make possible a stereoscopic 3D view and interaction with objects [14]. Head-mounted displays and intuitive handheld controllers provide users with flexibility and immersion for approaching the multidimensional anatomy of the patient in ways that would otherwise be difficult or impossible.

Cross-sectional viewing or 3D reconstructions viewed from 2D screens cannot provide a similar high level of immersion and authenticity as achievable with state-of-theart VR systems. Thus, VR may be a preferable and faster method for learning and understanding of the anatomy of the organ being investigated $[15,16]$. In addition, the VR environment allows users to perceive critical anatomical landmarks and their spatial relationships in the same virtual space, which may lead to better memory recall as compared to a traditional 2D screen interface [17].

A thorough understanding of the complex anatomy of the temporal bone (TB) and the lateral skull base is a lifelong endeavor and is considered as one of the most complex anatomical regions in humans. Indeed, it poses a formidable challenge even for experienced otologic surgeons, and thus it is not surprising that novice level surgeons often experience significant difficulties in acquiring an adequate understanding of the 3D characteristics of this anatomical entity.

In a recent study, expert otologic surgeons compared the VR environment with conventional cross-sectional visualization in TBs in a simulated preoperative setting; VR was found feasible for evaluating TB anatomy with good accuracy and reproducibility [18]. The aim of the present study was to compare the VR environment to a traditional cross-sectional viewing in a simulated preoperative planning setting by either expert or novice surgeons. We investigated the impact of the viewing method on the understanding of anatomy, the accuracy of measurements and the identification of anatomical landmarks in TB anatomy. Furthermore, we studied the subjective validity and evaluated the performance of participants with the two methods in both groups.

The study's hypothesis was that the VR environment does not offer additional benefits for the evaluation of TB anatomy compared to traditional cross-sectional (2D) viewing with equal subjective validity. In addition, we hypothesized that experienced surgeons will perform all identification and measurement tasks more accurately and faster compared to novices.

\section{Materials and methods}

\section{Ethics and permissions}

The study fulfilled the Helsinki Declaration for Ethical use of human material. It has an institutional approval (No. 125/2019), and the National Supervisory Authority for Welfare and Health authorized the use of cadaveric TBs (No. 9202/06.01.03.01/2013). The anonymity of participants was guaranteed, and informed consent was obtained from all participants.

\section{Data collection}

Five fresh-frozen TBs were harvested, and five 3-mm titanium screws fiducials were placed on each TB [18]. Three of the screws placed in the outer cortex and two in petrosal part served as fixed measurement points. A standard Vernier caliper (accuracy $0.02 \mathrm{~mm}$ ) under a surgical microscope was used to obtain the direct physical measurements (DPM) of the distances between the fiducials. TBs used in this study did not have any pre-existing pathology (e.g., malformations).

A Siemens SOMATOM Definition Flash (Siemens Healthcare, Forchheim, Germany) was used for high resolution computed tomography (HRCT) images $(120 \mathrm{kV}$, $96 \mathrm{mAs}$, FOV $85 \mathrm{~mm}$, pitch 0.8, CTDIvol $21.1 \mathrm{mGy}$, and $0.4 \mathrm{~mm}$ slice thickness). The adequacy of the HRCT quality for conventional cross-sectional viewing and VR environment, was evaluated by an experienced neuroradiologist (A.L.) and two experienced otologic surgeons (M.I-M., A.D.).

The images were viewed as 3D volumes in the VR environment and as cross-sectional viewing in a picture archiving and communication system (PACS) interface. The 3D model from the HRCT data in the VR environment was created with the Adesante SurgeryVision ${ }^{\mathrm{TM}}$ (Adesante Oy, Turku, Finland) medical software. A head-mounted display (HTC Vive Pro, HTC, New Taipei, Taiwan) with a pair of controllers was used to visualize and manipulate the 3D models in the VR environment generated by the software (Fig. 1). The cross-sectional HRCT image visualization was performed in our clinical PACS (Sectra AB, Linkoping, Sweden) in a 2D screen interface.

\section{Participants}

Two groups, based on their experience in otosurgery, were created: an expert group of five experienced otologic 

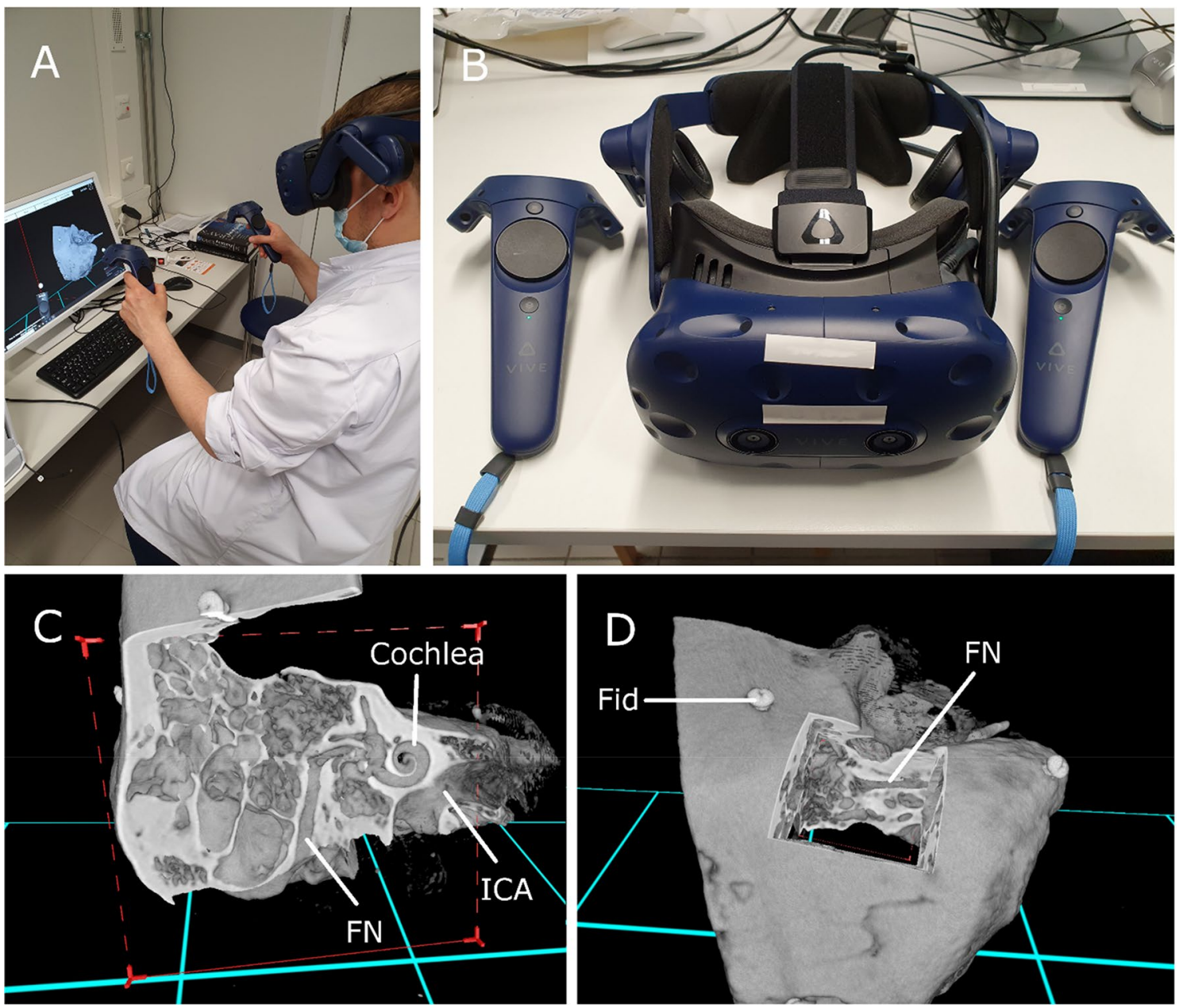

Fig. 1 a Setup of the VR interface during task performance, (b) Head-mounted display and a pair of controllers, (c) and (d) View of a temporal bone in the VR environment. $F N=$ Facial nerve, $I C A=$ Internal carotid artery, $F i d=$ Screw fiducial

surgeons and a novice group of four residents and one neurosurgeon with no experience in TB surgery.

\section{Tasks}

The same TBs and distance measurements as well as questionnaire data of the experts were used in our previous feasibility study [18]. In the present study, the experts were further asked to identify 24 anatomical landmarks (see Online Resource 1.). The same tasks i.e., identification of the anatomical landmarks, measuring the 11 distances between the surgically relevant anatomical structures and the 10 distances between the fiducials (see Online Resource 2.) were performed by novices for the same TBs in both VR and cross-sectional viewing. The time used by every participant for the completion of task was measured for each TB and method.

Before starting, each participant had a standardized 15-min familiarization session with the VR and PACS interfaces. The tasks were performed in a predetermined order. The five TBs were evaluated in a randomized order, first in VR and after minimum of 14 day period (mean $175 \mathrm{~d}$ with the expert group and $170 \mathrm{~d}$ with the novice group) in the PACS interface. This was done to minimize possible benefits for the VR environment, that could be acquired if the cross-sectional viewing was done first. The participants were allowed to adjust the image size, contrast, and brightness to their preferred setting. In addition, three preset windows optimized in the VR for bone, soft tissue and a translucent bone visualization were available. Linked multiplanar 2D reconstructions were used to make the measurements with the PACS interface. The participants were able to rotate the view and the planes in any direction to optimize the image sections for the measurements. The tasks were supervised by a senior otolaryngologist (T.T.). 


\section{Questionnaire}

After completion of the tasks, the participants evaluated the user experience and subjective validity of both methods with a modified 5-point Likert questionnaire including 20 domains (see Online Resource 3.). A free text section was added to highlight possible advantages and problems.

\section{Statistical analysis}

All of the statistical analyses, throughout the study, were supervised by a professional statistician and performed with IBM SPSS statistics version 27 (IBM SPSS, SPSS Inc., Chicago, IL, USA). The level of significance was set to $p<0.05$.

Inter-quartal range (IQR) was calculated for the distance measurements. Linear mixed model was used to compare the time spent in performing the tasks. The comparison of the landmark identification and measurement errors between the methods and between the groups was performed with Poisson loglinear test. Questionnaire results were compared with Wilcoxon signed rank test.

Intraclass correlation coefficient (ICC) was used as a measure of the correlation between the distances measured with different methods with the inter-rater reliability describing the agreement between different participants. Intra-rater reliability could not be tested, since the participants measured the TBs only once with each method.

The comparison of the anatomical measurements between the methods was calculated as a percentage difference to the respective median of the anatomical measurements conducted by the participants in each group.

\section{Results}

The demographics and surgical experience are summarized in Table 1. Two novice participants had experience in cadaver TB dissections (one time for both) before task completion. Of the novices with prior experience with the VR, one had trained surgical planning once with a skull model and other had trained the use of the software, head-mounted display, and controllers on a few occasions. None of the experts had prior experience with the VR before the study.

There were some missing anatomical measurements due to a partially fractured bony ear canal in one TB. The missing measurements, five in the novice and eleven in the expert group, were excluded from the analysis. A summary of all measurements and statistics is presented in Table 2 .

\section{Landmark identification}

The novice group correctly identified $95.3 \%$ and $92.8 \%$ of the landmarks in VR and cross-sectional viewing, respectively. The corresponding percentages for the expert group were $97.3 \%$ and $96.2 \%$. Experts made significantly fewer errors in cross-sectional viewing than novices $(p=0.016)$. No statistically significant difference was found in the VR interpretation between both groups $(p=0.070)$. The comparisons and statistics between the methods and groups are presented in Table 2.

\section{Fiducial and anatomical measurements}

A strong correlation between VR and cross-sectional viewing was found for the measurements of the fiducial $\left(\mathrm{ICC}_{\text {novices }} \geq 0.906, \mathrm{ICC}_{\text {experts }} \geq 0.916\right)$ and the anatomical distances $\left(\mathrm{ICC}_{\text {novices }} \geq 0.783, \mathrm{ICC}_{\text {experts }} \geq 0.900\right)$ in both groups. High inter-rater reliability (mean ICC $>0.848$ ) was found in both cross-sectional viewing and VR methods. The mean ICCs describing inter-rater reliability of the fiducial distance measurements performed in cross-sectional viewing and VR, respectively, were 0.920 and 0.963 in the novice group and 0.930 and 0.999 in the expert group. For the anatomical distance measurements, the respective mean ICCs were 0.848 and 0.925 in the novice group and 0.914 and 0.955 in the expert group.

The statistical comparisons of the methods and groups of the measurement errors are summarized in Table 2. Twentytwo (76\%) fiducial distance measurement errors were made by novices and fourteen (54\%) by experts in cross-sectional viewing; these were likely due to a misidentification of the fiducial measurement points (measured distance matched to
Table 1 Summary of participants' demographics. The values are medians (ranges) unless otherwise indicated

\begin{tabular}{lll}
\hline & Novice group $N=5$ & Expert group $N=5$ \\
\hline Age & $35(30-40)$ & $41(34-61)$ \\
Years of ENT or skull base experience, $y$ & $4.5(2-10)$ & $16.5(5.5-34)$ \\
Otological operations per year ${ }^{\mathrm{a}}$ & $1.5(0-6)$ & $72(48-120)$ \\
Sex male/female & $3 / 2$ & $4 / 1$ \\
Prior experience of VR technology & $2 / 5$ & $0 / 5$
\end{tabular}

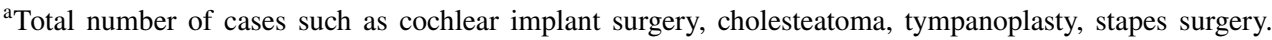
Smaller operations e.g., insertion of ventilation tubes excluded

${ }^{\mathrm{b}}$ Two novice participants had used VR only on a few occasions 
Table 2 Identification and measurement errors between (a) methods and (b) groups

\begin{tabular}{|c|c|c|c|c|c|c|}
\hline \multicolumn{7}{|c|}{ A. NOVICES } \\
\hline Error type & Method & Mean (SD) [\%] & $95 \% \mathrm{CI}[\%]$ & $\begin{array}{l}\text { Mean difference } \\
{[\%]^{\mathrm{a}}}\end{array}$ & $95 \% \mathrm{CI}$ difference $[\%]^{\mathrm{a}}$ & $p$ value $^{\mathrm{a}}$ \\
\hline \multirow[t]{2}{*}{ Landmarks } & PACS & $1.43(1.19)$ & $0.94,1.93$ & 0.52 & $-0.04,1.07$ & 0.066 \\
\hline & VR & $0.93(0.65)$ & $0.67,1.20$ & & & \\
\hline \multirow[t]{2}{*}{ Fiducials } & PACS & $2.32(2.29)$ & $1.38,3.26$ & 1.09 & $0.01,2.17$ & $0.049^{*}$ \\
\hline & VR & $1.36(2.81)$ & $0.20,2.52$ & & & \\
\hline \multirow[t]{2}{*}{ Anatomical } & PACS & $4.00(1.96)$ & $3.19,4.81$ & 0.10 & $-1.40,1.58$ & 0.903 \\
\hline & VR & $3.93(2.39)$ & $2.94,4.91$ & & & \\
\hline \multirow[t]{2}{*}{ Total } & PACS & $2.26(1.07)$ & $1.82,2.70$ & 0.52 & $-0.01,1.04$ & 0.054 \\
\hline & VR & $1.76(1.08)$ & $1.32,2.21$ & & & \\
\hline \multicolumn{7}{|c|}{ A. EXPERTS } \\
\hline Error type & Method & Mean (SD) [\%] & $95 \% \mathrm{CI}[\%]$ & $\begin{array}{l}\text { Mean difference } \\
{[\%]^{\mathrm{a}}}\end{array}$ & $95 \% \mathrm{CI}$ difference $[\%]^{\mathrm{a}}$ & $p$ value $^{\mathrm{a}}$ \\
\hline \multirow[t]{2}{*}{ Landmark } & PACS & $0.77(0.41)$ & $0.60,0.94$ & 0.29 & $-0.12,0.70$ & 0.166 \\
\hline & VR & $0.53(0.47)$ & $0.34,0.73$ & & & \\
\hline \multirow[t]{2}{*}{ Fiducials } & PACS & $2.08(3.19)$ & $0.76,3.40$ & 1.65 & $0.22,3.09$ & $0.024 *$ \\
\hline & VR & $0.40(0.82)$ & $0.06,0.74$ & & & \\
\hline \multirow[t]{2}{*}{ Anatomical } & PACS & $1.96(1.81)$ & $1.22,2.71$ & -2.36 & $-3.68,-1.04$ & $<0.001^{*}$ \\
\hline & VR & $4.22(2.39)$ & $3.23,5.20$ & & & \\
\hline \multirow[t]{2}{*}{ Total } & PACS & $1.35(0.98)$ & $0.95,1.76$ & -0.09 & $-0.53,0.34$ & 0.672 \\
\hline & VR & $1.40(0.64)$ & $1.14,1.67$ & & & \\
\hline \multicolumn{7}{|l|}{ B. PACS } \\
\hline Error type & Group & Mean (SD) [\%] & $95 \% \mathrm{CI}[\%]$ & $\begin{array}{l}\text { Mean difference } \\
{[\%]^{\mathrm{a}}}\end{array}$ & $95 \%$ CI difference $[\%]^{\mathrm{a}}$ & $p$ value $^{\mathrm{a}}$ \\
\hline \multirow[t]{2}{*}{ Landmark } & Novices & $1.43(1.19)$ & $0.94,1.93$ & 0.66 & $0.12,1.18$ & $0.016^{*}$ \\
\hline & Experts & $0.77(0.41)$ & $0.60,0.94$ & & & \\
\hline \multirow[t]{2}{*}{ Fiducial } & Novices & $2.32(2.29)$ & $1.38,3.26$ & 0.46 & $-0.76,0.17$ & 0.464 \\
\hline & Experts & $2.08(3.19)$ & $0.76,3.40$ & & & \\
\hline \multirow[t]{2}{*}{ Anatomical } & Novices & $4.00(1.96)$ & $3.19,4.81$ & 2.19 & $0.89,3.49$ & $0.001^{*}$ \\
\hline & Experts & $1.96(1.81)$ & $1.22,2.71$ & & & \\
\hline \multirow[t]{2}{*}{ Total } & Novices & $2.26(1.07)$ & $1.82,2.70$ & 0.95 & $0.45,1.45$ & $<0.001^{*}$ \\
\hline & Experts & $1.35(0.98)$ & $0.95,1.76$ & & & \\
\hline \multicolumn{7}{|l|}{ B. VR } \\
\hline Error type & Group & Mean (SD) [\%] & $95 \% \mathrm{CI}[\%]$ & $\begin{array}{l}\text { Mean difference } \\
{[\%]^{\mathrm{a}}}\end{array}$ & $95 \%$ CI difference $[\%]^{\mathrm{a}}$ & $p$ value $^{\mathrm{a}}$ \\
\hline \multirow[t]{2}{*}{ Landmark } & Novices & $0.93(0.65)$ & $0.67,1.20$ & 0.40 & $-0.33,0.83$ & 0.070 \\
\hline & Experts & $0.53(0.47)$ & $0.34,0.73$ & & & \\
\hline \multirow[t]{2}{*}{ Fiducial } & Novices & $1.36(2.81)$ & $0.20,2.52$ & 1.02 & $-0.31,2.34$ & $0.011^{*}$ \\
\hline & Experts & $0.40(0.82)$ & $0.06,0.74$ & & & \\
\hline \multirow[t]{2}{*}{ Anatomical } & Novices & $3.93(2.39)$ & $2.94,4.91$ & -0.26 & $-1.77,1.25$ & 0.734 \\
\hline & Experts & $4.22(2.39)$ & $3.23,5.20$ & & & \\
\hline \multirow[t]{2}{*}{ Total } & Novices & $1.76(1.08)$ & $1.32,2.21$ & 0.34 & $-0.13,0.80$ & 0.154 \\
\hline & Experts & $1.40(0.64)$ & $1.14,1.67$ & & & \\
\hline
\end{tabular}

Values are proportions \% unless otherwise indicated

In the landmark identification task, a misidentified anatomical landmark was classified as an identification error. The measurements outside of 1.5 IQRs were determined as outliers and counted as measurement errors in fiducial and anatomical measurements. Errors were converted into percentages per participant to allow a better comparison of task performance with each method

$V R$ virtual reality environment, PACS conventional cross-sectional (2D) method

${ }^{\text {a}}$ Poisson loglinear test

*Statistically significant difference between the methods 
a distance between some other fiducial pair, i.e., not the correct pair). The corresponding errors in VR were $9(53 \%)$ and 0 in the novice and expert group, respectively.

\section{Errors in total}

Statistical analysis of all errors revealed that the novice group made significantly more errors with cross-sectional viewing than the expert group (127 errors vs. 76 errors, $p<0.001$ ), whereas with VR, the difference between the groups remained statistically insignificant (Table 2). In total, the novices made fewer errors with VR than with crosssectional viewing (99 errors vs. 127 errors, $p=0.054$ ). In the expert group, the number of errors was not method dependent $(p=0.672)$.

When the first TB task was compared to the last, the number of errors in total were found to decrease in both groups with both methods. In the expert group, the mean difference of errors between first and last TB was $2.67 \%(p=0.273)$ in VR and $4.00 \%(p=0.095)$ in cross-sectional viewing. In the novice group, the corresponding values were $3.56 \%$ $(p=0.170)$ in VR and $0.89 \%(p=0.793)$ in cross-sectional viewing. A summary of the overall error development is presented in Fig. 2.

\section{Time needed for task completion}

The statistics and development of time used for task completion are illustrated in Fig. 2; Table 3. The experts were significantly faster when using the cross-sectional viewing in PACS interface than novices, but with VR, the difference between the groups remained statistically insignificant.

In the comparison of the time development between the first and the last TB task, the mean difference of time (minutes) used in novice group was 35.27 in cross-sectional viewing $(p<0.001)$ and 29.12 in VR $(p<0.001)$. In the expert group, the corresponding values were $14.00(p=0.009)$ and $37.99(p<0.001)$.

\section{Questionnaire}

The scores for the questionnaire are illustrated in Table 4 . The mean overall scores for VR were significantly better than those for cross-sectional viewing in both novice and expert groups $(p<0.001)$.

An analysis of the free text feedback revealed that VR provided a better 3D understanding of the anatomical structures and their relationship. The freedom to approach the target from any direction and angle in the VR environment was
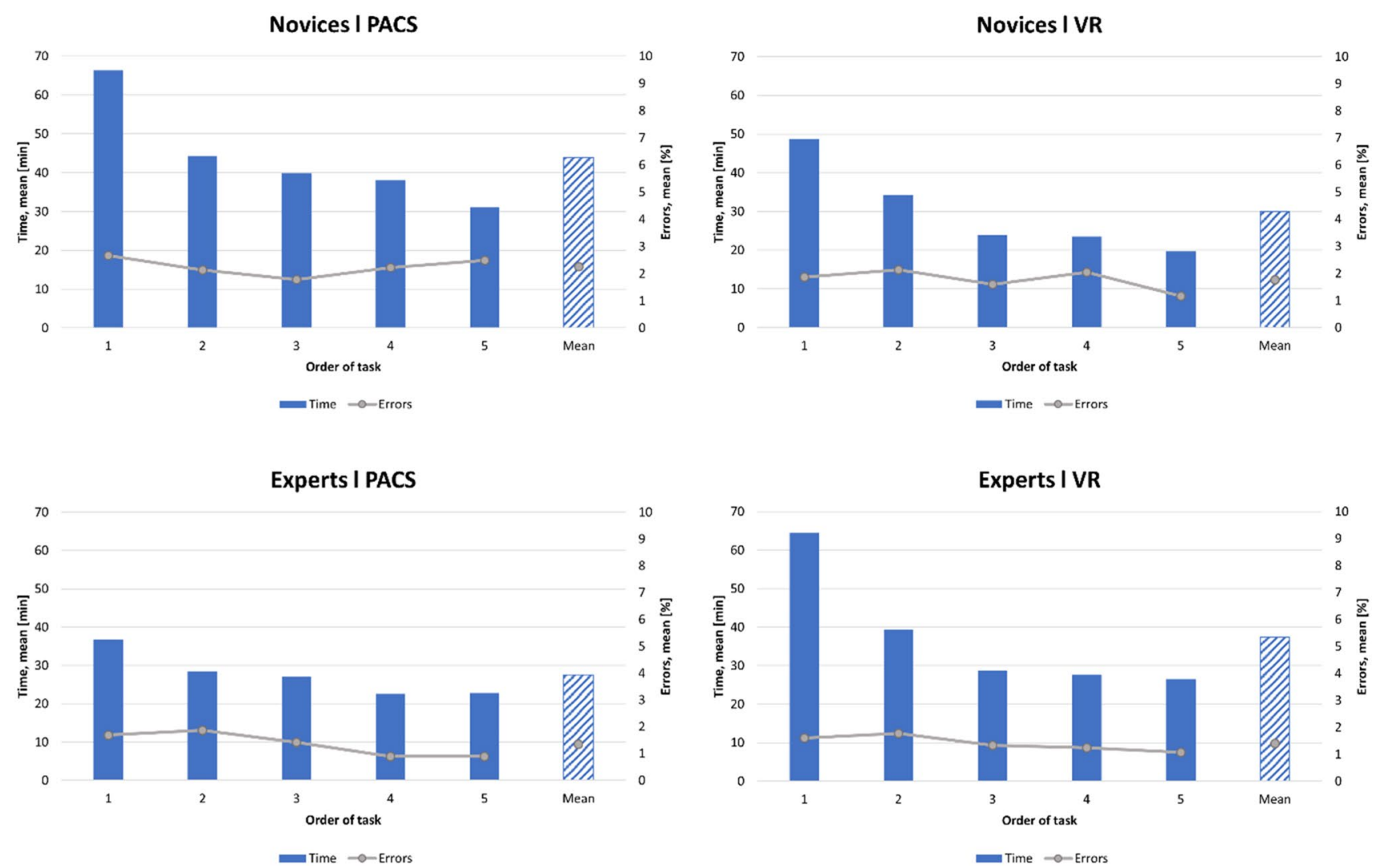

Fig. 2 Mean time used and number of errors (\%) with TB task progression in both study groups 
Table 3 Duration of tasks between methods and groups. All values are times (min) unless otherwise indicated

\begin{tabular}{lllllll}
\hline & Method/Group & Mean (SD) [min] & $95 \%$ CI [min] & $\begin{array}{l}\text { Mean differ- } \\
\text { ence [min] }]^{\mathrm{a}}\end{array}$ & $\begin{array}{l}95 \% \text { CI mean } \\
\text { difference } \\
{[\mathrm{min}]^{\mathrm{a}}}\end{array}$ & $p$ value $^{\mathrm{a}}$ \\
\hline Novices & PACS & $43.89(21.86)$ & $37.04,50.74$ & 13.85 & $4.96,22.74$ & $0.003^{*}$ \\
& VR & $30.04(15.17)$ & $6.68,53.39$ & & & \\
Experts & PACS & $27.53(11.79)$ & $20.68,34.38$ & -9.81 & $-17.72,-1.90$ & $0.016^{*}$ \\
& VR & $37.34(18.43)$ & $13.98,60.67$ & & & \\
PACS & NOVICES & $43.89(21.86)$ & $28.68,59.09$ & 16.36 & $6.64,26.04$ & $0.001^{*}$ \\
& EXPERTS & $27.53(11.79)$ & $16.99,38.07$ & & & \\
VR & NOVICES & $30.04(15.17)$ & $14.83,45.24$ & -7.03 & $-40.34,25.73$ & 0.665 \\
& EXPERTS & $37.34(18.43)$ & $26.80,47.88$ & & & \\
\hline
\end{tabular}

$V R=$ virtual reality environment, $P A C S=$ conventional cross-sectional (2D) method

${ }^{a}$ Linear mixed model test

*Statistically significant difference between the methods

Table 4 Responses to the questionnaire

\begin{tabular}{|c|c|c|c|c|c|c|}
\hline & \multicolumn{2}{|l|}{ Novices } & \multirow[t]{2}{*}{$p$ value $^{\mathrm{a}}$} & \multicolumn{2}{|l|}{ Experts } & \multirow[t]{2}{*}{$p$ value $^{\mathrm{a}}$} \\
\hline & VR (SD) & PACS (SD) & & VR (SD) & PACS (SD) & \\
\hline Appearance of anatomical structures & $4.6(0.5)$ & $3.2(0.4)$ & 0.059 & $4.6(0.5)$ & $3.4(0.5)$ & $0.034 *$ \\
\hline Appearance of tools & $3.4(0.5)$ & $3.6(1.0)$ & 0.785 & $3.2(0.4)$ & $3.2(0.7)$ & 1.000 \\
\hline Usability of tools & $3.4(0.8)$ & $3.2(0.7)$ & 0.705 & $3.0(0.6)$ & $3.0(1.1)$ & 1.000 \\
\hline Performance of tools & $4.0(0.6)$ & $3.4(1.2)$ & 0.334 & $3.8(0.4)$ & $3.4(0.5)$ & 0.157 \\
\hline Haptic feedback & $3.2(1.3)$ & $2.4(1.2)$ & 0.414 & $3.6(0.5)$ & $3.0(0.6)$ & 0.830 \\
\hline Ergonomics & $4.0(0.9)$ & $3.6(0.5)$ & 0.414 & $3.2(0.4)$ & $3.6(1.0)$ & 0.317 \\
\hline Depth perception & $4.6(0.8)$ & $2.0(0.6)$ & 0.059 & $4.4(0.8)$ & $2.0(0.9)$ & $0.034 *$ \\
\hline Quality of graphics & $4.2(0.7)$ & $3.2(0.7)$ & 0.180 & $4.4(0.8)$ & $4.0(0.9)$ & 0.317 \\
\hline Learning of anatomy & $4.8(0.4)$ & $3.2(0.7)$ & $0.039^{*}$ & $4.6(0.5)$ & $3.2(0.4)$ & 0.059 \\
\hline Learning of surgical planning & $4.6(0.8)$ & $2.8(1.2)$ & 0.066 & $4.6(0.5)$ & $3.4(0.5)$ & 0.063 \\
\hline Understanding of anatomical structures & $4.8(0.4)$ & $3.2(1.2)$ & $0.034 *$ & $4.4(0.5)$ & $3.4(0.5)$ & $0.034^{*}$ \\
\hline Quality of measuring anatomical structures & $4.4(0.5)$ & $3.2(1.5)$ & 0.336 & $4.4(0.5)$ & $2.8(0.7)$ & 0.059 \\
\hline $\begin{array}{l}\text { Understanding the relationships of anatomical } \\
\text { structures }\end{array}$ & $4.8(0.4)$ & $2.8(1.0)$ & $0.041^{*}$ & $4.6(0.5)$ & $3.0(0.6)$ & 0.180 \\
\hline Accuracy of measurement tool & $4.4(0.8)$ & $4.0(0.9)$ & 0.414 & $4.0(0.9)$ & $3.6(0.8)$ & 0.577 \\
\hline Hand-eye-coordination & $4.4(0.8)$ & $3.2(1.0)$ & 0.141 & $3.8(0.4)$ & $3.0(0.0)$ & $0.046^{*}$ \\
\hline Overall score for surgical planning & $4.4(0.5)$ & $3.2(1.0)$ & 0.109 & $4.2(0.4)$ & $3.4(0.5)$ & 0.102 \\
\hline \multicolumn{7}{|l|}{ Global rating } \\
\hline Recommend to colleague & $4.6(0.5)$ & $3.8(1.5)$ & 0.414 & $4.6(0.5)$ & $4.2(0.7)$ & 0.157 \\
\hline User-friendly & $4.4(0.5)$ & $3.0(1.1)$ & 0.059 & $3.6(0.8)$ & $3.6(1.0)$ & 1.000 \\
\hline Inclusion to surgical planning & $4.6(0.5)$ & $4.0(1.1)$ & 0.414 & $4.6(0.5)$ & $4.8(0.4)$ & 0.317 \\
\hline Understanding of the surgical site & $4.8(0.4)$ & $3.4(1.0)$ & 0.066 & $4.6(0.5)$ & $3.8(0.4)$ & $0.046^{*}$ \\
\hline Mean overall scores & $4.3(0.8)$ & $3.2(1.1)$ & $<0.001 *$ & $4.1(0.8)$ & $3.4(0.9)$ & $<0.001 *$ \\
\hline
\end{tabular}

All values are nominal (SD) on a 5-point Likert scale unless otherwise indicated. Score 1 represented not true/realistic/useful and 5 represented very true/realistic/useful. A score of 3 was considered neutral

$V R=$ virtual reality environment, $P A C S=$ conventional cross-sectional (2D) method, $S D=$ standard deviation

${ }^{a}$ Wilcoxon signed rank test

*Statistically significant difference between the methods 
much appreciated. All participants rated the VR as an excellent tool for learning anatomy and for preoperative planning.

\section{Discussion}

Previous studies have demonstrated the feasibility and effectiveness of exploiting 3D techniques and VR environments in teaching anatomy, especially for medical students [19-21]. For example, in reconstructive surgery, there are several studies demonstrating that virtual surgical planning may improve surgical accuracy and clinical outcomes [22-24]. Studies focusing on TB anatomy have evaluated the feasibility of VR for different simulator platforms (i.e., mastoidectomy training) targeted for surgical training and skill assessment $[25,26]$. To the best of our knowledge, there are only a few studies that have investigated the effect of VR technology on learning the anatomy of TB in residents and experienced otosurgeons [27, 28]. Therefore, we felt it would be interesting to examine the impact of VR for anatomy understanding and on performance in different anatomical tasks in a simulated preoperative planning setting in TBs.

Previously, it has been demonstrated that in experienced surgeons, the VR environment can provide equally accurate results as compared to conventional cross-sectional viewing [18]. Our present study explored the effect of a VR environment designed for surgery planning with respect to measurement accuracy, identification of anatomical structures, participants performance evaluating TB anatomy, and subjective validity of the TB's complex anatomy in novice and experienced surgeons.

We detected strong correlations between all distance measurements for each method in the novice group supporting the validity of measurements in VR. The time needed to complete the tasks and the numbers of errors demonstrated that the expert group was significantly faster and more accurate than the novice group when making the measurements with the cross-sectional views. In addition, this study revealed a rather long learning curve of the novice participants with cross-sectional viewing of complex anatomical structures compared to that in the VR. Our results demonstrate that two-dimensional images are appropriate and adequate for pre-operative planning for surgeons with prior experience and pre-existing anatomic knowledge gained from continuous surgical training and clinical work. In addition, expert surgeons are also trained to use cross-sectional viewing with PACS interface in preoperative planning and, therefore, possess better skills and ability to mentally reconstruct the cross-sectional data into a complex 3D model. For novices, however, our results show that VR viewing may convey additional information, which they can obviously utilize for a faster apprehension of the complex TB anatomy.
Interestingly, the 3D model readily created in the VR environment appeared to provide a better foundation for understanding complex anatomical structures, such as the TB, since the VR method may not require as much mental $3 \mathrm{D}$ reconstruction experience as the cross-sectional viewing with PACS. This argument can be supported by the following findings: with the VR, the novices had a lower number of errors for every task (landmark identifications, fiducial, and anatomical measurements) and significantly better time consumption results as compared to the cross-sectional viewing. A rather compelling finding was that there were no significant differences between experts and novices in time consumption or the number of errors in VR environment and thus refuting our second hypothesis. This is of special importance considering the fact that the difference of errors in the expert group remained insignificant between VR and cross-sectional viewing. In addition, with VR, the total number of errors decreased more in the novice than in the expert group, and the novices' results in VR moved closer to the expert level as the tasks progressed from first to fifth TB, indicating a potentially shorter learning curve as compared to cross-sectional viewing. These findings demonstrate that VR may be advantageous especially for novice surgeons to acquire an in-depth 3D understanding of complex anatomical structure.

The results of subjective validity contradict with our hypothesis, as both groups favored the VR environment over cross-sectional viewing. This result may be due to the authenticity that was provided by VR. The results also demonstrate the surgeons' positive attitude for adopting VR into anatomy learning, training and preoperative use irrespective of their level of experience, which is consistent with previous VR simulator studies found in literature $[25,29]$. The novices rated VR higher (and cross-sectional viewing lower) than the expert group. This may reflect the fact that they are more dependent on the additional information provided by VR. In addition, they may be more interested in exploiting new technologies in their training and clinical use in comparison to experienced surgeons. However, the experts also favored VR over cross-sectional viewing.

Several studies have demonstrated that there is extensive variability in the spatial comprehension of individuals, which may affect learning of anatomy and eventually their abilities to perform the kinds of tasks demanded in this study $[30,31]$. Since both groups had one participant that made a substantial amount of incorrect fiducial measurements, it is assumed that this applies also to the participants of this study. However, it has also been demonstrated that visual 3D models could improve learning of anatomy in individuals with poor spatial abilities [32].

Although, we used the term "learning" on several occasions, we are aware of the fact that learning is a broader concept and thus not completely accurate, since this study 
focused on the evaluation of a novel system for presenting complex anatomy. However, for novices we observed improvements in performance with VR (less errors in less time), which represented undoubtedly a learning effect. Therefore, we think, the intuitive 3D representation of VR may enhance also learning.

\section{Limitations}

This study has certain limitations that require attention. The number of participants was small. With respect to the anatomical identifications and measurements, the large number of measurements points (1125 per group) ensured adequate statistical power. Due to the high inter-rater reliability, it can be argued that a larger number of participants would not have changed the results. Although it has been shown that there is a considerable individual variety in spatial comprehension abilities, it was beyond the scope of our study to test each participant's talents. However, including these individual skills in future trials would be beneficial to obtain a more detailed evaluation of the role of VR in anatomy learning and operative planning. Furthermore, the impact of VR technology on the surgical performance and clinical outcomes as well as surgery training (e.g., mastoidectomy training) was not investigated here and will be a topic to be evaluated in the future.

\section{Conclusion}

In the VR environment, novices were faster and committed fewer errors than in traditional cross-sectional viewing. In addition, novices' task performance approached the level of expert otosurgeons after only five TB VR sessions, indicating a potentially shorter learning curve for novices in evaluating temporal bone anatomy compared to cross-sectional viewing and supporting efficiency of the VR environment for evaluating complex TB anatomy. For experts, no difference in task performance between VR and cross-sectional viewing was found. Surgical experience of experts may effectively compensate the more limited information of crosssectional viewing; however, as novices, they also appeared to appreciate the availability of a 3D representation of $\mathrm{TB}$ anatomy.

Supplementary Information The online version contains supplementary material available at https://doi.org/10.1007/s00405-021-07183-9.

Acknowledgements The authors want to thank Tuomas Selander MSc., for his assistance in the statistical analyses. We are grateful to Ewen MacDonald for his careful revision of the English language. We also want to thank Sini Varonen MD, Antti Ruuskanen MD, Timo Karjalainen and Jyrki Tervaniemi MDD for their participation in this study.
Author contributions TT, AD, A-PE, HL, and MI-M conceptualized and designed the study. TT, AL, and MI-M collected the data. TT and MI-M analyzed the data. AD, PL, AL, HL, and A-PE contributed to analysis refinement. TT wrote the first draft of the manuscript. All authors substantially contributed to final data synthesis and in revising the final manuscript. All authors gave final approval to the submitted manuscript and are accountable for all aspects of the work.

Funding Open access funding provided by University of Eastern Finland (UEF) including Kuopio University Hospital. The study was funded by the Academy of Finland (A.D. Grant No. 333525), State Research Funding of the Kuopio University Hospital (A.D. Grant No. 5551853), the Finnish ORL-HNS Foundation (T.T.) and North Savo Regional Fund (T.T. Grant No. 65202121, A.D. Grant No. 65202054). The research infrastructure (Kuopio Microsurgery Center) was supported by the Regional Council of Pohjois-Savo and the European Regional Development funds.

Data availability The datasets used and/or analysed during the current study are available from the corresponding author on reasonable request.

Code availability Not applicable.

\section{Declarations}

Conflict of interest The authors have no conflict of interest to disclose.

Ethics approval The study fulfilled the Helsinki Declaration for Ethical use of human material. It has an institutional approval (No. 125/2019), and the National Supervisory Authority for Welfare and Health authorized the use of cadaveric TBs (No. 9202/06.01.03.01/2013).

Consent to participate Informed consent was obtained from all participants.

Open Access This article is licensed under a Creative Commons Attribution 4.0 International License, which permits use, sharing, adaptation, distribution and reproduction in any medium or format, as long as you give appropriate credit to the original author(s) and the source, provide a link to the Creative Commons licence, and indicate if changes were made. The images or other third party material in this article are included in the article's Creative Commons licence, unless indicated otherwise in a credit line to the material. If material is not included in the article's Creative Commons licence and your intended use is not permitted by statutory regulation or exceeds the permitted use, you will need to obtain permission directly from the copyright holder. To view a copy of this licence, visit http://creativecommons.org/licenses/by/4.0/.

\section{References}

1. Triepels CPR, Smeets CFA, Notten KJB, Kruitwagen RFPM, Futterer JJ, Vergeldt TFM et al (2020) Does three-dimensional anatomy improve student understanding? Clin Anat 33(1):25

2. Tomlinson SB, Hendricks BK, Cohen-Gadol A (2019) Immersive three-dimensional modeling and virtual reality for enhanced visualization of operative neurosurgical anatomy. World Neurosurg 01(131):313-320

3. Bergman EM, Der Vleuten V, Cees P, Scherpbier M, Albert JJA (2011) Why don't they know enough about anatomy? A narrative review. Med Teach 33(5):403-409 
4. Hoffman H, Vu D (1997) Virtual reality: teaching tool of the twenty-first century? Acad Med 72(12):1076-1081

5. Lee C, Wong GKC (2019) Virtual reality and augmented reality in the management of intracranial tumors: a review. J Clin Neurosci 62:14-20

6. Maresky HS, Oikonomou A, Ali I, Ditkofsky N, Pakkal M, Ballyk B (2019) Virtual reality and cardiac anatomy: exploring immersive three-dimensional cardiac imaging, a pilot study in undergraduate medical anatomy education. Clin Anat 32(2):238-243

7. Yammine K, Violato C (2015) A meta-analysis of the educational effectiveness of three-dimensional visualization technologies in teaching anatomy. Anatomical sciences education. Anat Sci Educ $8(6): 525$

8. Gnanasegaram JJ, Leung R, Beyea JA (2020) Evaluating the effectiveness of learning ear anatomy using holographic models. J Otolaryngol Head Neck Surg 49(1):63

9. Seixas-Mikelus SA, Adal A, Kesavadas T, Baheti A, Srimathveeravalli G, Hussain A et al (2010) Can image-based virtual reality help teach anatomy? J Endourol 24(4):629-634

10. Tan S, Hu A, Wilson T, Ladak H, Haase P, Fung K (2012) Role of a computer-generated three-dimensional laryngeal model in anatomy teaching for advanced learners. J Laryngol Otol 126(4):395-401

11. Chan S, Li P, Locketz G, Salisbury K, Blevins NH (2016) Highfidelity haptic and visual rendering for patient-specific simulation of temporal bone surgery. Comput Ass Surg 21(1):85-101

12. Zhao J, Xu X, Jiang H, Ding Y (2020) The effectiveness of virtual reality-based technology on anatomy teaching: a meta-analysis of randomized controlled studies. BMC Med Educ 20(1):127

13. Wainman B, Wolak L, Pukas G, Zheng E, Norman GR (2018) The superiority of three-dimensional physical models to twodimensional computer presentations in anatomy learning. Med Educ 52(11):1138-1146

14. Chinnock C (1994) Virtual reality in surgery and medicine. Hosp Technol 13(18):1

15. Solyar A, Cuellar H, Sadoughi B, Olson TR, Marvin P (2008) Endoscopic Sinus Surgery Simulator as a teaching tool for anatomy education. Am J Surg 196(1):120-124

16. Ellington DR, Shum PC, Dennis EA, Willis HL, Szychowski JM, Richter HE (2019) Female pelvic floor immersive simulation: a randomized trial to test the effectiveness of a virtual reality anatomic model on resident knowledge of female pelvic anatomy. $\mathrm{J}$ Minim Invasive Gynecol 26(5):897-901

17. Krokos E, Plaisant C, Varshney A (2019) Virtual memory palaces: immersion aids recall. Virtual Reality 23(1):1-15

18. Timonen T, Iso-Mustajarvi M, Linder P, Lehtimaki A, Lopponen H, Elomaa AP, et al. (2020) Virtual reality improves the accuracy of simulated preoperative planning in temporal bones: a feasibility and validation study. Eur Arch Otorhinolaryngol

19. Nicholson DT, Chalk C, Funnell WR, Daniel SJ (2006) Can virtual reality improve anatomy education? A randomised controlled study of a computer-generated three-dimensional anatomical ear model. Med Educ 40(11):1081-1087
20. Chen S, Zhu J, Cheng C, Pan Z, Liu L, Du J et al (2020) Can virtual reality improve traditional anatomy education programmes? A mixed-methods study on the use of a 3D skull model. BMC Med Educ 20(1):395

21. Yi-Chun Du, Fan S-C, Yang L-C (2020) The impact of multiperson virtual reality competitive learning on anatomy education: a randomized controlled study. BMC Med Educ 20(1):1-343

22. Ujiie H, Yamaguchi A, Gregor A, Chan H, Kato T, Hida Y et al (2021) Developing a virtual reality simulation system for preoperative planning of thoracoscopic thoracic surgery. J Thorac Dis 13(2):778-783

23. Witjes MJH, Schepers RH, Kraeima J (2018) Impact of 3D virtual planning on reconstruction of mandibular and maxillary surgical defects in head and neck oncology. Curr Opin Otolaryngol Head Neck Surg 26(2):108-114

24. Shenaq DS, Matros E (2018) Virtual planning and navigational technology in reconstructive surgery. J Surg Oncol 118(5):845-852

25. Andersen SAW, Varadarajan VV, Moberly AC, Hittle B, Powell KA, Wiet GJ (2021) Patient-specific virtual temporal bone simulation based on clinical cone-beam computed tomography. Laryngoscope

26. Fang TY, Wang PC, Liu CH, Su MC, Yeh SC (2014) Evaluation of a haptics-based virtual reality temporal bone simulator for anatomy and surgery training. Comput Methods Programs Biomed 113(2):674-681

27. Sethia R, Wiet G (2015) Preoperative preparation for otologic surgery. Curr Opin Otolaryngol Head Neck Surg 23(5):355-359

28. Morone PJ, Shah KJ, Hendricks BK, Cohen-Gadol AA (2019) Virtual, 3-dimensional temporal bone model and its educational value for neurosurgical trainees. World Neurosurg 01(122):e1412-e1415

29. Arora A, Khemani S, Tolley N, Singh A, Budge J, Varela DA, Diaz V et al (2012) Face and content validation of a virtual reality temporal bone simulator. Otolaryngol Head Neck Surg 146(3):497-503

30. Garg AX, Norman G, Sperotable L (2001) How medical students learn spatial anatomy. Lancet 357(9253):363-364

31. Lufler RS, Zumwalt AC, Romney CA, Hoagland TM (2012) Effect of visual-spatial ability on medical students' performance in a gross anatomy course. Anat Sci Educ 5(1):3-9

32. Berney S, Bétrancourt M, Molinari G, Hoyek N (2015) How spatial abilities and dynamic visualizations interplay when learning functional anatomy with $3 \mathrm{D}$ anatomical models: interplay of spatial ability and dynamic visualization. Anat Sci Educ $8(5): 452-462$

Publisher's Note Springer Nature remains neutral with regard to jurisdictional claims in published maps and institutional affiliations. 